\title{
Two-year outcomes of ab interno trabeculectomy with the Trabectome for Chinese primary open angle glaucoma: a retrospective multicenter study
}

\author{
Ya-Long Dang ${ }^{1,2,3,4}$, Xiao Wang ${ }^{1}$, Wan-Wei Dai ${ }^{1,2,5}$, Ping Huang ${ }^{1}$, Nils A Loewen ${ }^{3}$, Chun Zhang ${ }^{1,2}$, \\ China Trabectome Study Group, International Trabectome Study Group
}

${ }^{1}$ Department of Ophthalmology, Peking University Third Hospital, Beijing 100191, China

${ }^{2}$ Beijing Key Laboratory of Restoration of Damaged Optic Nerve, Peking University Third Hospital, Beijing 100191, China

${ }^{3}$ Department of Ophthalmology, University of Pittsburgh, Pittsburgh, Pennsylvania 15213, USA

${ }^{4}$ Department of Ophthalmology, Sanmenxia Central Hospital, Sanmenxia 472000, Henan Province, China

${ }^{5}$ Institution of Population Research, Peking University, Beijing 100191, China

Co-first authors: Ya-Long Dang and Xiao Wang

Correspondence to: Chun Zhang. 49 North Garden Road, Haidian District, Beijing 100191, China. zhangc1@yahoo.com Received: 2017-12-18Ａccepted: 2018-04-28

\footnotetext{
Abstract

- AIM: To evaluate the 2-year efficacy and safety of ab interno trabeculectomy with the Trabectome in Chinese primary open angle glaucoma (POAG) patients.

- METHODS: This was a multicenter, retrospective, observational study and included POAG patients with or without visually-significant cataracts. The Chinese patients were enrolled from three glaucoma centers and a group of comparable Japanese POAG patients was analyzed from our international Trabectome database. The patients received Trabectome or a combined surgery with phacoemulsification and intraocular lens implantation. The primary outcome was intraocular pressure (IOP) reduction. Secondary outcomes included reduction of glaucoma medications, surgical complications, and success at $2 y$. Success was defined as: 1) IOP $\leq 21 \mathrm{~mm} \mathrm{Hg}$ and at least $20 \%$ IOP reduction from baseline after $3 \mathrm{mo}$ at any two consecutive visits; 2) no additional glaucoma surgery required.

- RESULTS: A total of $\mathbf{4 2}$ Chinese POAG patients from three glaucoma centers were enrolled. Twelve patients underwent Trabectome surgery combined with phacoemulsification and intraocular lens implantation while the remainder underwent Trabectome surgery alone. Thirteen patients had a history of failed glaucoma surgery and were
}

considered as complicated cases. In China data, the mean preoperative IOP was $21.4 \pm 1.23 \mathrm{~mm} \mathrm{Hg}$. The Trabectome lowered IOP to $17.9 \pm 1.8 \mathrm{~mm} \mathrm{Hg}$ at $2 \mathrm{y}(P=0.05)$. The number of glaucoma medications also decreased significantly from a baseline of $2.0 \pm 0.9$ to $1.1 \pm 0.8$ at $2 y$ post-surgery $(P=0.04)$. The overall 2 -year success rate was $78 \%$, with patients undergoing combined surgery having a higher success rate compared with those undergoing Trabectome surgery alone $(100 \%$ vs $76 \%)$. In Japan data, the mean preoperative IOP was $20.8 \pm 7.7 \mathrm{~mm} \mathrm{Hg}$. The Trabectome lowered IOP to $12.20 \pm 2.0 \mathrm{~mm} \mathrm{Hg}$ at $2 \mathrm{y}$. The number of glaucoma medications also decreased significantly from a baseline of $2.1 \pm 0.9$ to $3.4 \pm 0.6$ at $2 y$ post-surgery. In all patients, no major complications were seen.

- CONCLUSION: Surgery with the Trabectome appears to be an efficient and safe procedure in Chinese POAG patients in the long-term.

- KEYWORDS: primary open angle glaucoma; minimally invasive glaucoma surgeries; intraocular pressure; surgical complications

DOI:10.18240/ijo.2018.06.08

Citation: Dang YL, Wang X, Dai WW, Huang P, Loewen NA, Zhang C, China Trabectome Study Group, International Trabectome Study Group. Two-year outcomes of ab interno trabeculectomy with the Trabectome for Chinese primary open angle glaucoma: a retrospective multicenter study. Int J Ophthalmol 2018;11(6):945-950

\section{INTRODUCTION}

D rimary open angle glaucoma (POAG) is a leading cause of irreversible blindness ${ }^{[1]}$, affecting more than 40 millions of people worldwide ${ }^{[2]}$. Intraocular pressure (IOP) is the only modifiable risk factor for $\mathrm{POAG}^{[3]}$. Traditional trabeculectomy and tube-shunt surgery efficiently reduce IOP in patients with moderate to advanced glaucoma but have a high incidence of complications ${ }^{[4-5]}$ and surgical failure in the long-term ${ }^{[6]}$. In contrast, microincisional glaucoma surgery provides a safe IOP reduction with a short recovery time ${ }^{[7]}$ has become an ideal option for early to moderate POAG ${ }^{[8]}$. 
Most minimally invasive glaucoma surgeries can be combined with cataract surgery through an ab interno approach. These procedures reduce IOP by different mechanisms: 1) ablate or bypass the trabecular meshwork (TM) using instruments such as the Trabectome (Neomedix Corporation, Tustin, CA, USA) ${ }^{[9-11]}$, iStent (Glaukos Corp, Laguna Hills, CA, USA) ${ }^{[12]}$, or the Hydrus mini-stent (Ivantis Inc., Irvine, CA, USA) ${ }^{[13]}$; 2) establish a new route for suprachoroidal drainage (CyPass Micro-Stent; Transcend Medical, Menlo Park, CA, USA $)^{[14]}$; 3 ) reduce the production of aqueous humor ${ }^{[15]}$; or 4) create a subconjunctival filtration route ${ }^{[16]}$. Developed by Neomedix (Tustin, CA, USA) more than a decade ago ${ }^{[9]}$, Trabectome ablates 60-120 degrees of TM and the inner wall of Schlemm's canal by high-frequency electrosurgery ${ }^{[17]}$. Trabectome provides active irrigation and aspiration, which maintains better stability and temperature in the anterior chamber, compared with passive chamber management ${ }^{[18]}$. No hypotony or bleb-related complications occur ${ }^{[10-11,19-20]}$.

First introduced in China in 2013, Trabectome shows promise for open angle glaucoma patients ${ }^{[19,21]}$. Our previous study suggested that Trabectome surgery significantly reduced IOP from a baseline of $22.5 \pm 8.1 \mathrm{~mm} \mathrm{Hg}$ to $17.6 \pm 6.4 \mathrm{~mm} \mathrm{Hg}$ at $1 \mathrm{y}$ postoperatively ${ }^{[19]}$, while the number of glaucoma medications was reduced by $40.0 \%$ with no major complications ${ }^{[19]}$. However, in that study, we included different types of openangle glaucomas, which might have different responses ${ }^{[10,22]}$. Also, the follow-up in that study was only 1y. The aim of the current study was to evaluate the 2-year efficacy and safety of $a b$ interno trabeculectomy with the Trabectome in Chinese POAG patients.

\section{SUBJECTS AND METHODS}

This was a multicenter, retrospective, observational study approved by the Institutional Review Board of Peking University Third Hospital in compliance with the Declaration of Helsinki. The need for patient informed consent was waived. POAG patients undergoing Trabectome surgery included those with or without visually-significant cataract except those who: 1) had concurrent keratitis, conjunctivitis or uveitis in the study eye; 2) had other abnormalities preventing reliable applanation tonometry, and had a follow-up of less than 3 mo.

Trabectome surgery was performed as we described previously ${ }^{[10,19]}$. Briefly, a 1.6-mm iris parallel clear corneal incision was made at the temporal side. After the insertion of the Trabectome tip into the anterior chamber, the eye was pressurized with active irrigation. The goniolens was then placed on the cornea, and the TM was engaged with the instrument at a $45^{\circ}$ upward angle to facilitate the best visualization to the surgeon. A total of $120^{\circ}-180^{\circ}$ of the nasal TM was ablated for each patient. After removal of the TM debris, a few viscoelastic materials were injected to minimize postoperative hyphema. The patients with visually significant cataracts received phacoemulsification with intraocular lens implantation immediately after Trabectome. The patients were followed up at $1 \mathrm{~d}, 1 \mathrm{wk}, 1,3,6,12,18$, and 24mo. IOP was measured by Goldmann applanation tonometry. The target IOP was determined individually by the treating physician. Patients with a visually-significant cataract underwent phacoemulsification after the Trabectome surgery. Visual field defects were categorized as mild, moderate, and advanced based on Humphrey visual field testing defined as by HodappParrish-Anderson criteria ${ }^{[23]}$. Gonioscopy, Snellen visual acuity, and stereoscopic optic nerve evaluation were performed routinely before surgery.

The primary outcome was IOP reduction, while the secondary outcomes included reduced number of glaucoma medications, surgical complications, and 2-year success rate. Success was defined as: 1) IOP $<21 \mathrm{~mm} \mathrm{Hg}$ and at least $20 \%$ IOP reduction from baseline after $3 \mathrm{mo}$ at any two consecutive visits; 2) no additional glaucoma surgery required.

Statistical Analysis Quantitative data were presented as mean \pm standard deviation and were statistically analyzed using R (statistical package version 3.0.0, Free Software Foundation, Boston, MA, USA) ${ }^{[24]}$. The Wilcoxon's test was used to compare postoperative IOPs and the number of glaucoma medications with their respective baseline values. We computed Kaplan-Meier curves and compared them using the log-rank test. We could not determine the risk factors associated with Trabectome surgery failure by logistic regression because of the small sample size. A $P$-value $\leq 0.05$ was considered statistically significant.

\section{RESULTS}

After excluding 2 cases without postoperative information, a total of 42 POAG patients from three independent glaucoma centers (Peking University Third Hospital, Beijing Tongren Hospital, and Peking University People's Hospital) were enrolled in this study. A group of comparable Japanese POAG patients was analyzed from our international Trabectome database. Patients' demographics and characteristics are summarized in Table 1. In China data, the mean age was $55 \pm 21 \mathrm{y}$, and most of patients were male. Half of the patients had preoperative best-corrected visual acuity better than 20/40; $76 \%$ of these patients $(n=32)$ were phakic and 12 underwent combined surgery of Trabectome and phacoemulsification. Thirteen patients with a history of previous failed glaucoma surgery were considered complicated cases and had previously undergone trabeculectomy $(n=10)$, or Trabectome $(n=2)$ or tube-shunt surgery ( $n=1$; Table 1$)$.

In China data, the mean preoperative IOP was $21.4 \pm 1.23 \mathrm{~mm} \mathrm{Hg}$, and at one year post-surgery, the mean value dropped to the lowest value of $16.5 \pm 2.8 \mathrm{~mm} \mathrm{Hg}(P<0.01$, compared with baseline). Then, mean IOP increased slightly but remained statistically lower than baseline value throughout the study 
Int J Ophthalmol， Vol. 11， No. 6, Jun.18, 2018 www.ijo.cn Tel:8629-82245172 8629-82210956 Email:ijopress@163.com

Table 1 Demographic of matched data

\begin{tabular}{|c|c|c|}
\hline Parameters & $\begin{array}{c}\text { China group } \\
(n=42)\end{array}$ & $\begin{array}{c}\text { Japan group } \\
(n=35)\end{array}$ \\
\hline Age, mean $\pm \mathrm{SD}$ (range), y & $55 \pm 21(19-87)$ & $63 \pm 9(21-92)$ \\
\hline Gender (F/M) & $11 / 31$ & $14 / 21$ \\
\hline \multicolumn{3}{|l|}{ Preop. Snellen acuity } \\
\hline$>20 / 20$ & $0(0)$ & $5(14)$ \\
\hline $20 / 20-20 / 40$ & $21(50)$ & $15(43)$ \\
\hline $20 / 50-20 / 70$ & $6(14)$ & $4(11)$ \\
\hline $20 / 80-20 / 100$ & $4(10)$ & $1(3)$ \\
\hline $20 / 200-20 / 400$ & $5(12)$ & $0(0)$ \\
\hline$<20 / 400$ & $1(2)$ & $0(0)$ \\
\hline NR & $6(14)$ & $10(29)$ \\
\hline \multicolumn{3}{|l|}{ VF } \\
\hline Mild & $6(14)$ & $3(9)$ \\
\hline Moderate & $9(21)$ & $11(31)$ \\
\hline Advanced & $11(26)$ & $10(29)$ \\
\hline NR & $16(38)$ & $11(31)$ \\
\hline \multicolumn{3}{|l|}{ Disc C/D } \\
\hline$<0.7$ & $6(14)$ & $4(11)$ \\
\hline 0.7 to 0.8 & $20(48)$ & $6(18)$ \\
\hline$>0.8$ & $13(31)$ & $11(31)$ \\
\hline NR & $3(7)$ & $14(40)$ \\
\hline \multicolumn{3}{|l|}{ Prior surgeries } \\
\hline SLT/ALT/LI/ECP & $0(0)$ & $0(0)$ \\
\hline Trabeculectomy/trabeculotomy & $10(24)$ & $3(9)$ \\
\hline Trabectome & $2(5)$ & $0(0)$ \\
\hline Shunt & $1(2)$ & $0(0)$ \\
\hline Retina surgery & $0(0)$ & $0(0)$ \\
\hline \multicolumn{3}{|l|}{ Combined surgeries } \\
\hline Trabectome + phaco & $12(29)$ & $12(34)$ \\
\hline Trabectome only & $30(71)$ & $23(66)$ \\
\hline
\end{tabular}

SD: Standard deviation; NR: Not recorded; VF: Visual field; SLT: Selective laser trabeculoplasty; ALT: Argon laser trabeculoplasty; IL: Laser iridectomy; ECP: Endoscopic cyclophotocoagulation.

(all comparisons, $P<0.05$; Table 2 ). Trabectome surgery also decreased patients' number of glaucoma medications from a baseline of $2.0 \pm 0.9$ to $1.1 \pm 0.9$ at one year. At the last followup at $24 \mathrm{mo}$, the number of glaucoma medications was still significantly lower than baseline ( $P=0.04$; Table 2$)$.

Based on the success criteria (IOP $\leq 21 \mathrm{~mm} \mathrm{Hg}$ and $>20 \%$ IOP reduction from baseline without additional glaucoma surgery), the overall success rate of China data was $78 \%$ at $24 \mathrm{mo}$. Chinese patients undergoing combined Trabectome surgery and phacoemulsification $(n=12)$ had a higher success rate than did those undergoing Trabectome surgery alone $(n=30$, $100 \%$ vs $76 \%$, respectively; Figure 1). Patients who underwent prior Trabectome surgery $(n=2)$ achieved their target IOP at 24 mo while $2 / 10$ patients undergoing previous trabeculectomy suffered surgical failure; one patient at $9 \mathrm{mo}$ and one at $15 \mathrm{mo}$.

In China group, by the end of the 24 mo follow-up, one patient had undergone repeat Trabectome surgery at 6 o'clock and
Table 2 Wilcoxon test were applied by comparing IOP and number of medications between two groups mean $\pm \mathrm{SD}(n)$

\begin{tabular}{|c|c|c|c|}
\hline Parameters & China group & Japan group & $P$ \\
\hline \multicolumn{4}{|l|}{ IOP } \\
\hline Baseline & $21.4 \pm 1.23(42)$ & $20.8 \pm 7.7(35)$ & \\
\hline $1 \mathrm{mo}$ & $17.8 \pm 6.7(41)$ & $14.5 \pm 4.2(34)$ & 0.01 \\
\hline $3 \mathrm{mo}$ & $17.0 \pm 3.8(23)$ & $14.1 \pm 4.1(28)$ & 0.01 \\
\hline $6 \mathrm{mo}$ & $17.1 \pm 3.2(20)$ & $13.8 \pm 3.6(20)$ & $<0.01$ \\
\hline $9 \mathrm{mo}$ & $16.7 \pm 2.5(19)$ & $13.6 \pm 3.5(17)$ & $<0.01$ \\
\hline $12 \mathrm{mo}$ & $16.5 \pm 2.8(17)^{\mathrm{a}}$ & $14.3 \pm 3.7(17)$ & 0.08 \\
\hline $18 \mathrm{mo}$ & $17.8 \pm 2.6(14)$ & $13.5 \pm 2.5(8)$ & $<0.01$ \\
\hline $24 \mathrm{mo}$ & $17.9 \pm 1.8(11)^{\mathrm{b}}$ & $12.0 \pm 2.0(5)$ & $<0.01$ \\
\hline \multicolumn{4}{|c|}{ No. of medication } \\
\hline Baseline & $2.0 \pm 0.9(42)$ & $2.1 \pm 0.9(35)$ & \\
\hline $1 \mathrm{mo}$ & $0.8 \pm 0.9(41)$ & $1.7 \pm 1.5(34)$ & $<0.01$ \\
\hline $3 \mathrm{mo}$ & $0.7 \pm 0.9(23)$ & $1.9 \pm 1.3(31)$ & $<0.01$ \\
\hline $6 \mathrm{mo}$ & $0.8 \pm 0.9(20)$ & $2.4 \pm 1.2(20)$ & $<0.01$ \\
\hline $9 \mathrm{mo}$ & $1.0 \pm 0.9(19)$ & $2.4 \pm 1.2(17)$ & $<0.01$ \\
\hline $12 \mathrm{mo}$ & $1.1 \pm 0.9(17)$ & $2.3 \pm 1.2(17)$ & $<0.01$ \\
\hline $18 \mathrm{mo}$ & $0.9 \pm 0.7(14)$ & $2.5 \pm 1.1(8)$ & $<0.01$ \\
\hline $24 \mathrm{mo}$ & $1.1 \pm 0.8(11)^{\mathrm{c}}$ & $3.4 \pm 0.6(5)$ & $<0.01$ \\
\hline
\end{tabular}

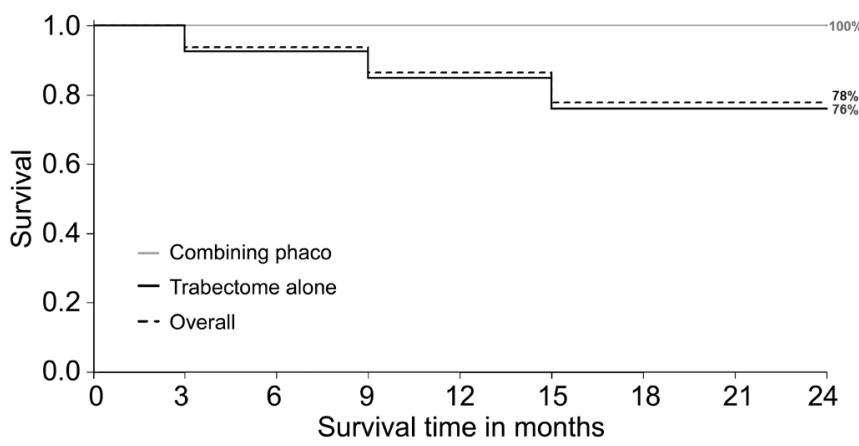

Figure 1 Two-year success rate Applying the success criteria, the overall success rate of China data was $78 \%$ at 24 mo. Kaplan-Meier curves were used for survival analysis.

four patients had undergone EX-PRESS (Alcon Laboratories, Inc., Fort Worth, TX, USA) shunt implantation. All patients had achieved their IOP goals at the last visit. No visionthreatening complications and bleb-related complications were encountered, and no patients experienced visual acuity loss of more than two lines.

\section{DISCUSSION}

TM is a complex multilayer structure that regulates aqueous outflow in a pressure dependent fashion ${ }^{[25]}$. In glaucomatous eyes, diseased TM has a reduced facility and shows reduced phagocytosis ${ }^{[26-27]}$, increased stiffness ${ }^{[28]}$, and stress fiber formation ${ }^{[27]}$. Either removing TM surgically ${ }^{[29]}$ or 
rehabilitating TM function by stem cells ${ }^{[30]}$ can restore normal outflow and decrease IOP in previous studies. In the current study, our results showed that ab interno trabeculectomy with the Trabectome efficiently reduced IOP and the number of glaucoma medications with an overall success rate of $78 \%$ during a 2-year follow-up. No major complications were seen highlighting the safety aspects of this procedure.

Open-angle glaucoma (except neovascular glaucoma) is the main indication for ab interno trabeculectomy with the Trabectome. A retrospective cohort study by Jordan et $a l^{[31]}$ showed that IOP decreased from a mean baseline of $24 \pm 5.5$ to $18 \pm 6.1 \mathrm{~mm} \mathrm{Hg} 204 \mathrm{~d}$ after Trabectome surgery for a consecutive 261 POAG patients. The authors' results were similar to our findings as well as those of a Meta-analysis by Chow et $a l^{[32]}$. In POAG, the Trabectome has comparable or better IOP-lowering effects on secondary open-angle glaucoma, depending on the preoperative IOP. Akil et $a l^{[22]}$ found that with similar preoperative values, IOP reduction in pigmentary glaucoma cases was similar to that in POAG. In our previous studies, patients with pseudoexfoliativeand steroid-induced glaucoma with higher preoperative IOP finally obtained more IOP reduction than age- and gendermatched POAG controls ${ }^{[10,33-34]}$. Even though preoperative IOP was higher in these patients, the overall success rate was unchanged ${ }^{[10,22,35-36]}$ or even better ${ }^{[35,37]}$ than for POAG patients. This is likely because the causes of secondary open-angle glaucoma are mostly TM-related diseases while POAG has a more complicated mechanism, including dysfunction of downstream outflow tracts.

In this study, 12 patients underwent Trabectome surgery combined with phacoemulsification. All of these patients achieved the success criteria at $2 \mathrm{y}$ follow-up, in contrast with only $76 \%$ in the Trabectome only group. This is consistent with previous findings by other groups showing that combined surgery has a lower risk of failure than for Trabectome alone ${ }^{[34,38]}$. However, it is worth noting that a higher success rate in patients undergoing combined surgery does not mean a greater IOP reduction. In fact, patients undergoing combined surgery consistently have less IOP reduction compared with the Trabectome only group because of the low preoperative IOP in the combined group ${ }^{[7,33-34,37-38]}$. A recent study by Tojo et $a l^{[38]}$ suggested that a low preoperative IOP and combined surgery contribute to success with the Trabectome.

Trabectome was once thought suitable only for mild or moderate glaucoma. Our previous studies indicated that patients with higher glaucoma severity achieved greater IOP reduction ${ }^{[11,39]}$. In the current study, we included 10 patients with advanced POAG and a history of previous trabeculectomy. Eight of these patients met the success criteria at the end of 24 mo follow-up. In another study, Wecker et $a l^{[40]}$ found that the Trabectome efficiently decreased IOP in patients with previously failed trabeculectomy from $24.5 \pm 3.5$ to $15.7 \pm 3.4 \mathrm{~mm} \mathrm{Hg}$ ( $36 \%$ reduction) over $415 \mathrm{~d}$ observation.

Reducing the number of medications is another benefit of Trabectome surgery; $0.99 \pm 0.54$ fewer medications are expected following Trabectome alone and $0.76 \pm 0.35$ fewer medications following Trabectome combined with phacoemulsification ${ }^{[7]}$. Consistent with our previous work ${ }^{[19]}$, the POAG patients in this study obtained an average of 0.8 fewer medications (40\% reduction) compared with baseline.

Similar to other microincisional glaucoma procedures, Trabectome surgery has a desirable safety profile. The most common complication is transient blood reflux ${ }^{[41]}$ and transient hypotony $(<5 \mathrm{~mm} \mathrm{Hg})^{[22]}$, which usually spontaneously resolves within a few days of surgery. Less than $1 \%$ visionthreatening complications were reported ${ }^{[7]}$, including one case of endophthalmitis ${ }^{[42]}$, and four cases of aqueous misdirection ${ }^{[29,31,43-44]}$. Previous studies suggested approximately $6.1 \%$ to $34.9 \%$ of patients required a secondary glaucoma surgery ${ }^{[35,37,40]}$. In this study, there was no bleb so bleb-related complications were avoided, and only five patients underwent additional glaucoma surgery; one patient underwent repeat Trabectome surgery and four underwent a lumenal control glaucoma filtration device (EX-PRESS, Alcon Laboratories, Inc., Fort Worth, TX, USA) implantation.

In this study, trabectome showed an effective and safe procedure in lowing IOP and glaucoma medication. Since glaucoma treatment is a balance between lowing IOP and reducing glaucoma medication taking. Based on this analysis, glaucoma practice in Japan tended to lower IOP in comparison to practice in China where glaucoma practice tended to lower the glaucoma medication.

Actually, this study has several limitations. First, the small sample size was insufficient to determine the risk factors associated with Trabectome failure by logistic regression. Second, we focused on IOP reduction but not on restoration or improved visual function. A recent study by Caprioli et $a l^{[45]}$ indicated that trabeculectomy might reverse the glaucomatous dysfunction of retinal ganglion cells and their central projections. In conclusion, our data suggested that ab interno trabeculectomy with the Trabectome is an efficient and safe procedure in Chinese POAG patients in the long-term. In future, a largesample-size clinical trial with more visual function evaluations are desired to address the limitations of our study.

\section{ACKNOWLEDGEMENTS}

Foundation: Supported by the National Natural Science Foundation of China (No.81670851).

Conflicts of Interest: Dang YL, None; Wang X, None; Dai WW, None; Huang P, None; Loewen NA has received honoraria for wet labs and lectures from Neomedix Inc.; Zhang C, None; China Trabectome Study Group, None; International Trabectome Study Group, None. 


\section{REFERENCES}

1 Cedrone C, Mancino R, Cerulli A, Cesareo M, Nucci C. Epidemiology of primary glaucoma: prevalence, incidence, and blinding effects. Prog Brain Res 2008;173:3-14.

2 Tham YC, Li X, Wong TY, Quigley HA, Aung T, Cheng CY. Global prevalence of glaucoma and projections of glaucoma burden through 2040: a systematic review and meta-analysis. Ophthalmology 2014;121(11):2081-2090.

3 Lusthaus JA, Goldberg I. Investigational and experimental drugs for intraocular pressure reduction in ocular hypertension and glaucoma. Expert Opin Inv Drugs 2016;25(10):1201-1208.

4 Razeghinejad MR, Havens SJ, Katz LJ. Trabeculectomy bleb-associated infections. Surv Ophthalmol 2017;62(5):591-610.

5 Borisuth NS, Phillips B, Krupin T. The risk profile of glaucoma filtration surgery. Curr Opin Ophthalmol 1999;10(2):112-116.

6 Casson R, Rahman R, Salmon JF. Long term results and complications of trabeculectomy augmented with low dose mitomycin $\mathrm{C}$ in patients at risk for filtration failure. Br J Ophthalmol 2001;85(6):686-688.

7 Kaplowitz K, Bussel II, Honkanen R, Schuman JS, Loewen NA. Review and meta-analysis of ab-interno trabeculectomy outcomes. $\mathrm{Br} J$ Ophthalmol 2016;100(5):594-600.

8 Kerr NM, Wang J, Barton K. Minimally invasive glaucoma surgery as primary stand-alone surgery for glaucoma. Clin Exp Ophthalmol 2017;45(4):393-400.

9 Francis BA, See RF, Rao NA, Minckler DS, Baerveldt G. Ab interno trabeculectomy: development of a novel device (Trabectome) and surgery for open-angle glaucoma. J Glaucoma 2006;15(1):68-73.

10 Dang Y, Kaplowitz K, Parikh HA, Roy P, Loewen RT, Francis BA, Loewen NA. Steroid-induced glaucoma treated with trabecular ablation in a matched comparison with primary open-angle glaucoma. Clin Exp Ophthalmol 2016;44(9):783-788.

11 Roy P, Loewen RT, Dang Y, Parikh HA, Bussel II, Loewen NA. Stratification of phaco-trabectome surgery results using a glaucoma severity index in a retrospective analysis. BMC Ophthalmol 2017;17(1):30.

12 Spiegel D, García-Feijoó J, García-Sánchez J, Lamielle H. Coexistent primary open-angle glaucoma and cataract: preliminary analysis of treatment by cataract surgery and the iStent trabecular micro-bypass stent. Adv Ther 2008;25(5):453-464.

13 Camras LJ, Yuan F, Fan S, Samuelson TW, Ahmed IK, Schieber AT, Toris CB. A novel Schlemm's canal scaffold increases outflow facility in a human anterior segment perfusion model. Invest Ophthalmol Vis Sci 2012;53(10):6115-6121.

14 Saheb H, Ianchulev T, Ahmed II. Optical coherence tomography of the suprachoroid after CyPass Micro-Stent implantation for the treatment of open-angle glaucoma. Br J Ophthalmol 2014;98(1):19-23.

15 Alvarado JA. Endocyclophotocoagulation for pediatric glaucoma: a tale of two cities. J AAPOS 2007;11(1):10-11.

16 Lewis RA. Ab interno approach to the subconjunctival space using a collagen glaucoma stent. J Cataract Refract Surg 2014;40(8):1301-1306.

17 Fallano K, Bussel I, Kagemann L, Lathrop KL, Loewen N. Training strategies and outcomes of ab interno trabeculectomy with the trabectome.
F1000Res 2017;6:67.

18 Wang C, Dang Y, Waxman S, Xia X, Weinreb RN, Loewen NA. Angle stability and outflow in dual blade ab interno trabeculectomy with active versus passive chamber management. PLoS One 2017;12(5):e0177238.

19 Huang P, Wang H, Wu H, Sun Y, Wang M, Cui Y, Wang N. Preliminary investigation on the safety and efficacy of Trabectome. Zhonghua Yan Ke Za Zhi 2015;51(2):115-119.

20 Bussel II, Kaplowitz K, Schuman JS, Loewen NA. Outcomes of ab interno trabeculectomy with the trabectome by degree of angle opening. Br J Ophthalmol 2015;99(7):914-919.

21 Lee JW, Yick DW, Tsang S, Yuen CY, Lai JS. Efficacy and safety of trabectome surgery in Chinese open-angle glaucoma. Medicine (Baltimore) 2016;95(15):e3212.

22 Akil H, Chopra V, Huang A, Loewen N, Noguchi J, Francis BA. Clinical results of ab interno trabeculotomy using the Trabectome in patients with pigmentary glaucoma compared to primary open angle glaucoma. Clin Exp Ophthalmol 2016;44(7):563-569.

23 Hodapp E, Parrish RK, Anderson DR. Clinical decisions in glaucoma. St Louis: Mosby. 1993;52-61.

24 Ihaka R, Gentleman R. R: a language for data analysis and graphics. $J$ Comput Graph Stat 1996;5(3):299-314.

25 Llobet A, Gasull X, Gual A. Understanding trabecular meshwork physiology: a key to the control of intraocular pressure? News Physiol Sci 2003;18:205-209.

26 Dang Y, Waxman S, Wang C, Loewen RT, Loewen NA. Intraocular pressure elevation precedes a phagocytosis decline in a model of pigmentary glaucoma. F1000Res 2018;7:174.

27 Lütjen-Drecoll E, Tektas OY. Structural changes in the trabecular meshwork with primary open angle glaucoma. Encyclopedia of the Eye 2010;214(5):224-228.

28 Wang K, Read AT, Sulchek T, Ethier CR. Trabecular meshwork stiffness in glaucoma. Exp Eye Res 2017;158:3-12.

29 Ahuja Y, Ma Khin Pyi S, Malihi M, Hodge DO, Sit AJ. Clinical results of ab interno trabeculotomy using the trabectome for openangle glaucoma: the Mayo Clinic series in Rochester, Minnesota. Am J Ophthalmol 2013;156(5):927-935.e2.

30 Zhu W, Gramlich OW, Laboissonniere L, Jain A, Sheffield VC, Trimarchi JM, Kuehn MH. Transplantation of iPSC-derived TM cells rescues glaucoma phenotypes in vivo. Proc Natl Acad Sci U S A 2016;113(25):E3492-E3500.

31 Jordan JF, Wecker T, van Oterendorp C, Anton A, Reinhard T, Boehringer D, Neuburger M. Trabectome surgery for primary and secondary open angle glaucomas. Graefes Arch Clin Exp Ophthalmol 2013;251(12):2753-2760.

32 Chow JTY, Hutnik CML, Solo K, Malvankar-Mehta MS. When is evidence enough evidence? A systematic review and meta-analysis of the trabectome as a solo procedure in patients with primary open-angle glaucoma. J Ophthalmol 2017;2017:2965725.

33 Dang Y, Roy P, Bussel II, Loewen RT, Parikh H, Loewen NA. Combined analysis of trabectome and phaco-trabectome outcomes by glaucoma severity. F1000Res 2016;5:762. 
34 Okeke CO, Miller-Ellis E, Rojas M. Trabectome success factors. Medicine (Baltimore) 2017;96(24):e7061.

35 Shoji N, Kasahara M, Iijima A, Takahashi M, Tatsui S, Matsumura

K, Shimizu K. Short-term evaluation of Trabectome surgery performed on Japanese patients with open-angle glaucoma. Jpn J Ophthalmol 2016;60(3):156-165.

36 Mizoguchi T, Nishigaki S, Sato T, Wakiyama H, Ogino N. Clinical results of Trabectome surgery for open-angle glaucoma. Clin Ophthalmol 2015;9:1889-1894.

37 Ting JL, Damji KF, Stiles MC. Ab interno trabeculectomy: outcomes in exfoliation versus primary open-angle glaucoma. $J$ Cataract Refract Surg 2012;38(2):315-323.

38 Tojo N, Abe S, Hayashi A. Factors that influence of trabectome surgery for glaucoma patients. J Glaucoma 2017;26(9):835-844.

39 Loewen RT, Roy P, Parikh HA, Dang Y, Schuman JS, Loewen NA. Impact of a glaucoma severity index on results of trabectome surgery: larger pressure reduction in more severe glaucoma. PLoS One 2016;11(3):e0151926.

40 Wecker T, Neuburger M, Bryniok L, Bruder K, Luebke J, Anton A,
Jordan JF. Ab interno trabeculectomy with the Trabectome as a valuable therapeutic option for failed filtering blebs. J Glaucoma 2016;25(9):758-762. 41 Hashemian SJ, Miraftabi A, Jafari ME, Hemami MR. Combined cataract extraction and trabeculotomy by the internal approach for coexisting cataract and open-angle glaucoma. J Curr Ophthalmol 2017;29(1):17-22.

42 Kaplowitz K, Chen X, Loewen N. Two year results for 180 degree trabectome ablation. San Francisco, CA: American Glaucoma Society Annual Meeting. Poster. 2013.

43 Mosaed S, Rhee DJ, Filippopoulos T, Tseng H, Deokule S, Weinreb RN. Trabectome outcomes in adult open-angle glaucoma patients: one-year follow-up. Clinical and Surgical Ophthalmology 2010;28(8-9): 182-186.

44 Minckler D, Mosaed S, Dustin L, Ms BF. Trabectome (trabeculectomyinternal approach): additional experience and extended follow-up. Trans Am Ophthalmol Soc 2008;106:149-159; discussion 159-160.

45 Caprioli J, de Leon JM, Azarbod P, Chen A, Morales E, NouriMahdavi K, Coleman A, Yu F, Afifi A. Trabeculectomy can improve longterm visual function in glaucoma. Ophthalmology 2016;123(1):117-128. 